SUBJECT AREAS:

THEORETICAL PHYSICS

BOSE-EINSTEIN CONDENSATES

Received

20 October 2014

Accepted

22 December 2014

Published

26 January 2015

Correspondence and requests for materials should be addressed to

Z.-D.H.

(huyuanda1112@

jiangnan.edu.cn)

\section{Spin and field squeezing in a spin-orbit coupled Bose-Einstein condensate}

\author{
Yixiao Huang' \& Zheng-Da Hu ${ }^{2}$
}

\author{
'Department of Physics and Center of Theoretical and Computational Physics, The University of Hong Kong, Hong Kong, China, \\ ${ }^{2}$ School of Science, Jiangnan University, Wuxi 214122 , China.
}

Recently, strong spin-orbit coupling with equal Rashba and Dresselhaus strength has been realized in neutral atomic Bose-Einstein condensates via a pair of Raman lasers. In this report, we investigate spin and field squeezing of the ground state in spin-orbit coupled Bose-Einstein condensate. By mapping the spin-orbit coupled BEC to the well-known quantum Dicke model, the Dicke type quantum phase transition is presented with the order parameters quantified by the spin polarization and occupation number of harmonic trap mode. This Dicke type quantum phase transition may be captured by the spin and field squeezing arising from the spin-orbit coupling. We further consider the effect of a finite detuning on the ground state and show the spin polarization and the quasi-momentum exhibit a step jump at zero detuning. Meanwhile, we also find that the presence of the detuning enhances the occupation number of harmonic trap mode, while it suppresses the spin and the field squeezing.

S pin-orbit coupling (SOC) describes an intrinsic interaction between the spin and the momentum of a particle. SOC plays a major role in condensed matter systems, such as spin and anomalous Hall effects ${ }^{1,2}$, topological insulators and topological superconductors ${ }^{3}$. Recently it is found that SOC also plays a key role in realizing spintronics ${ }^{4}$ and topological quantum computing ${ }^{5}$. However, the SOC physics in typical solid-state materials is hard to observe because the strength of the SOC is generally much smaller than the Fermi velocity of electrons. Quantum many-body systems of ultra-cold atom gases can be precisely controlled in experiments, and would thus provide an ideal platform to explore novel SOC physics and device applications ${ }^{6}$. Recently, SOC with equal Rashba and Dresselhaus strength has been realized in a neutral atomic Bose-Einstein condensate (BEC) by dressing two atomic spin states with a pair of Raman lasers ${ }^{7}$. Moreover, such SOC strength is much stronger than that in typical solid-state materials. With the strong strength of SOC, spins are not conserved during their motion and rich exotic superfluid phenomena have been revealed ${ }^{8-31}$. For instance, new topological excitations and new ground state phases have been observed in spin-orbit coupled Fermi gases and BEC, respectively ${ }^{32-38}$. Here we study the spin and the field (bosonic) squeezing in the system of ultracold atoms in the trapped BEC with the equal Rashba and Dresselhaus SOCs.

Spin squeezing ${ }^{39-42}$, arising from quantum correlations of collective spin systems, has potential applications in quantum information processes, quantum metrology and atom interferometers ${ }^{43,44}$. It is also closely related to quantum entanglement ${ }^{45-48}$. In the past few years, many efforts have been devoted to the generation of squeezing in atomic systems. In recent experiments, it has been reported that spin squeezing can be generated in a ensemble of atoms via atom-photon interactions or a BEC via atom collisions ${ }^{49-52}$.

In this work, we investigate properties of the ground state of a spin-orbit coupled BEC and show that both spin and field squeezing can be induced by the SOC in the BEC. First, we map the spin-orbit coupled BEC to the wellknown quantum Dicke model and show that there exists a quantum phase transition (QPT) from a superradiant (spin polarized) to normal (spin balanced) phase by tuning two Raman lasers with a current experimental setup of National Institute of Standards and Technology (NIST). In the superradiant phase, macroscopic spin polarization and occupation number of harmonic trap mode are presented. By contrast, in the normal phase, the values of spin polarization and occupation number of harmonic trap mode become zero. The behaviors of spin and field squeezing near the critical point are also explored. It is found that, at the critical point, the maximal spin squeezing is achieved and a sudden transition occurs for the field squeezing, which can indicate the QPT. In addition, the field squeezing is independent of the SOC strength in this phase. We also discuss the effects of the detuning on the ground state of spin polarization and quasi-momentum, and show the results agree with those in recent experiments $^{18}$. The presence of the detuning suppresses the spin and field squeezing, while it enhances the occupation number of harmonic trap mode. 


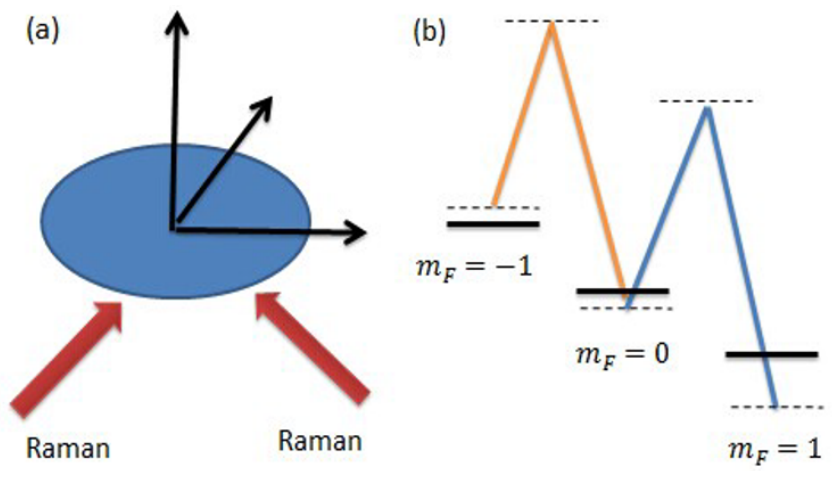

Figure 1 Illustration about the model and the level diagram. (a) The experimental scheme for realizing the equal Rashba and Dresselhaus SOC in the trapped BEC. (b) Level diagram: three hyperfine states $|-1\rangle,|0\rangle$, and $|1\rangle$ are coupled by the Raman lasers.

\section{Results}

Model and quantum phase transition. In a recent benchmark experiment, the equal Rashba and Dresselhaus SOCs were realized in the trapped BEC with ${ }^{87} \mathrm{Rb}$ atoms ${ }^{7}$, as illustrated in Fig. 1(a), all ultracold atoms are prepared in a two dimensional BEC in the $x y$ plane with a strong confinement along the $z$ axis. Such a two dimensional setup does not affect the essential physics, since the $z$ direction is decoupled with the SOC. With a large detuning $\Delta$ from the excited state, the hyperfine ground state $\left|F=1, m_{F}=-1\right\rangle$ and $\left|F=1, m_{F}=0\right\rangle$ can be defined as effective spin- $\uparrow$ and spin- $\downarrow$ respectively, which is shown in Fig.1 (b). In the dressed state basis $\left\{|\tilde{\uparrow}\rangle=\exp \left(i \mathbf{k}_{1} r\right)|\uparrow\rangle\right.$ and $\left.|\tilde{\downarrow}\rangle=\exp \left(i \mathbf{k}_{2} r\right)|\downarrow\rangle\right\}$ with $\mathbf{k}_{1}$ and $\mathbf{k}_{2}$ denoting the wave vectors of the Raman lasers, equal Rashba and Dresselhaus SOCs can be induced by two Raman lasers incident at a $\pi / 4$ angle from the $x$ axis and the corresponding dynamics of single particle of the BEC is governed by the following nonlinear GrossPitaevskii (GP) equation

$$
i \hbar \partial \Psi / \partial t=\left(p^{2} / 2 m+V(\mathbf{r})+H_{s}+H_{I}\right) \Psi,
$$

where $\Psi=\left(\boldsymbol{\psi}_{\uparrow}, \Psi_{\downarrow}\right)^{T}$ is the wavefunction on the dressed state basis and satisfies the normalization condition $\int d x d y\left(\left|\Psi_{\uparrow}\right|^{2}+\left|\Psi_{\downarrow}\right|^{2}\right)=1$, $p$ is the momentum, and $V(\mathbf{r})=\frac{m}{2}\left(\omega_{x}^{2} x^{2}+\omega_{y}^{2} y^{2}\right)$ is the harmonic trap potential with $m$ being the mass of the ultracold atom and $\omega_{x, y}$ denoting the trapping frequencies in the $x, y$ axes. In addition, the Hamiltonian $H_{s}$ describing the equal Rashba and Dresselhaus SOCs is given by

$$
H_{s}=\gamma p_{x} \sigma_{z}+\hbar \Omega \sigma_{x}+\frac{\delta}{2} \sigma_{z}
$$

where $\sigma_{x}$ and $\sigma_{z}$ are the Pauli matrices, $\gamma=\sqrt{2} \pi \hbar /(m \lambda)$ describes the SOC strength with $\lambda$ denoting the wave length of the Raman lasers, $\Omega$ measures the Raman coupling strength, and $\delta$ is the detuning from the level splitting. The many-body interaction Hamiltonian is $H_{I}=$ $\operatorname{diag}\left(g_{\uparrow \uparrow}\left|\Psi_{\uparrow}\right|^{2}+g_{\uparrow \downarrow}\left|\Psi_{\downarrow}\right|^{2}, g_{\uparrow \downarrow}\left|\Psi_{\uparrow}\right|^{2}+g_{\downarrow \downarrow}\left|\Psi_{\downarrow}\right|^{2}\right)$, where $g_{\uparrow \uparrow}=g_{\uparrow \downarrow}=$ $4 \pi \hbar^{2} N\left(c_{0}+c_{2}\right) /\left(m a_{z}\right)$ and $g_{\downarrow \downarrow}=4 \pi \hbar^{2} N c_{0} /\left(m a_{z}\right)$ represent the inter- and intra-spin collision interactions, respectively. Here, $c_{0}$ and $c_{2}$ are the $s$-wave scattering lengths, $N$ is the atom number, and $a_{z}=\sqrt{2 \pi \hbar / m \omega_{z}}$ with the trapping frequency $\omega_{z}$ in the $z$ axis.

In the present experiment, the $s$-wave scattering lengths have been measured as $c_{0}=100.86 a_{B}$ and $c_{2}=-0.46 a_{B}$, where $a_{B}$ is the Bohr radius. Then we can find $g_{\uparrow \uparrow}=g_{\uparrow \downarrow} \approx g_{\downarrow \downarrow}$. Introducing the harmonic mode operators, $a=\sqrt{m \omega_{x} / 2 \hbar}\left(x+i p_{x} / m \omega_{x}\right)$ and $b=\sqrt{m \omega_{y} / 2 \hbar}$ $\left(y+i p_{y} / m \omega_{y}\right)$, the Hamiltonian (1) for $N$ particles can be mapped to a Dicke type model ${ }^{16-18}$

$$
\begin{aligned}
H_{T} & =\hbar \omega_{y} N b^{\dagger} b+\hbar \omega_{x} N a^{\dagger} a+\hbar \Omega S_{x}+\frac{\delta}{2} S_{z} \\
& +\gamma \sqrt{2 m \hbar \omega_{x}} i\left(a^{\dagger}-a\right) S_{z},
\end{aligned}
$$

where $S_{z}=\sum_{i}^{N} \sigma_{i, z} / 2$ and $S_{x}=\sum_{i}^{N} \sigma_{i, x} / 2$ are the collective spin operators. Since the boson mode in the $y$ direction does not interact with the ultracold atom, the system can be reduced to

$$
\begin{aligned}
H_{T} & =\hbar \omega_{x} N a^{\dagger} a+\hbar \Omega S_{x}+\frac{\delta}{2} S_{z} \\
& +\gamma \sqrt{2 m \hbar \omega_{x}} i\left(a^{\dagger}-a\right) S_{z},
\end{aligned}
$$

When $\delta=0$, the Hamiltonian is equivalent to the standard Dicke model $^{53}$. Here, we shall note that if the repulsive interaction between the different spin components is sufficiently strong, the mapping to the Dicke model is invalid, which is due to the fact that the trapped BEC is unstable when $g_{\uparrow \downarrow} \gg\left\{g_{\uparrow \uparrow}, g_{\downarrow \downarrow}\right\}$. In contrast, if there is no interaction between the trapped BEC, there will be no correlation between the ultracold atoms and all of the ultracold atoms will occupy both $K_{\min }= \pm \sqrt{\gamma^{2}-\Omega^{2} / 4 \gamma^{2}}$ in the momentum space. Then, the single spatial mode approximation which we have used is not valid.

For the system with Hamiltonian (4), there is a quantum phase transition between the normal phase and the superradiant phase by changing the Raman coupling strength $\Omega$. By means of the mean field coherent state approach, the critical point for the phase transition of the system can be obtained. We assume the wave function of the ground state is given by $|\psi\rangle=|\theta\rangle:|\alpha\rangle$, where $|\theta\rangle$ is the spin coherent state and $|\alpha\rangle$ is the spatial coherent state with $a|\alpha\rangle=\alpha|\alpha\rangle$ and $\alpha=u$ $+i v$. In the absence of the detuning term, i.e., $\delta=0$, the energy of the system is (with the constant dropped)

$$
\begin{aligned}
E & =N \hbar \omega_{x}\left(u^{2}+v^{2}\right)-N \hbar k_{R} \sqrt{\frac{2 \hbar \omega_{x}}{m}} v \cos \theta \\
& +\frac{\Omega}{2} N \hbar \sin \theta .
\end{aligned}
$$

Minimizing the energy $E$ with respect to $\theta$, the critical point is given by $\Omega_{c}=4 E_{R} / \hbar$, where $E_{R}=\hbar^{2} k_{R}^{2} / 2 m$ is the recoil energy. When $\Omega>\Omega_{c}$, the system is in the spin-balanced normal phase, where the spin polarization and the occupation number of harmonic trap mode are zero. In the region $\Omega<\Omega_{\mathcal{c}}$, the ground state has two degenerate local minima with spin polarization $\left\langle S_{z}\right\rangle= \pm \frac{N}{2} \sqrt{1-\left(\Omega / \Omega_{c}\right)^{2}}$ and the occupation number of harmonic trap mode $\bar{n}_{p}=\frac{\hbar k_{R}^{2}}{2 m \omega_{x}}\left[1-\left(\Omega / \Omega_{c}\right)^{2}\right]$. As is shown in Fig. 2, the spin polarization $\left\langle S_{z}\right\rangle$ and rescaled occupation number of harmonic trap mode $N_{p}=2 m \omega_{x} \bar{n}_{p} /\left(\hbar k_{R}^{2}\right)$ as functions of $\Omega$ are plotted in the resonance case $(\delta=0)$. The direct numerical results respect to Hamiltonian (4) agree well with those by the mean field approximation. In the superradiant phase, the system has a macroscopic occupation number of harmonic trap mode and spin polarization which coincides well with the results in the recent experiment ${ }^{18}$. In contrast, in the normal phase, there are no occupation number of harmonic trap mode and spin polarization. As a consequence, either the spin polarization or the occupation number of harmonic trap mode can be considered as an order parameter of QPT.

Spin and field squeezing. Now we discuss the applications of the spin-orbit coupled BECs in quantum information science. Spin squeezing has potential application in atom interferometers and high precision atom clocks. It can also signify long-range quantum 

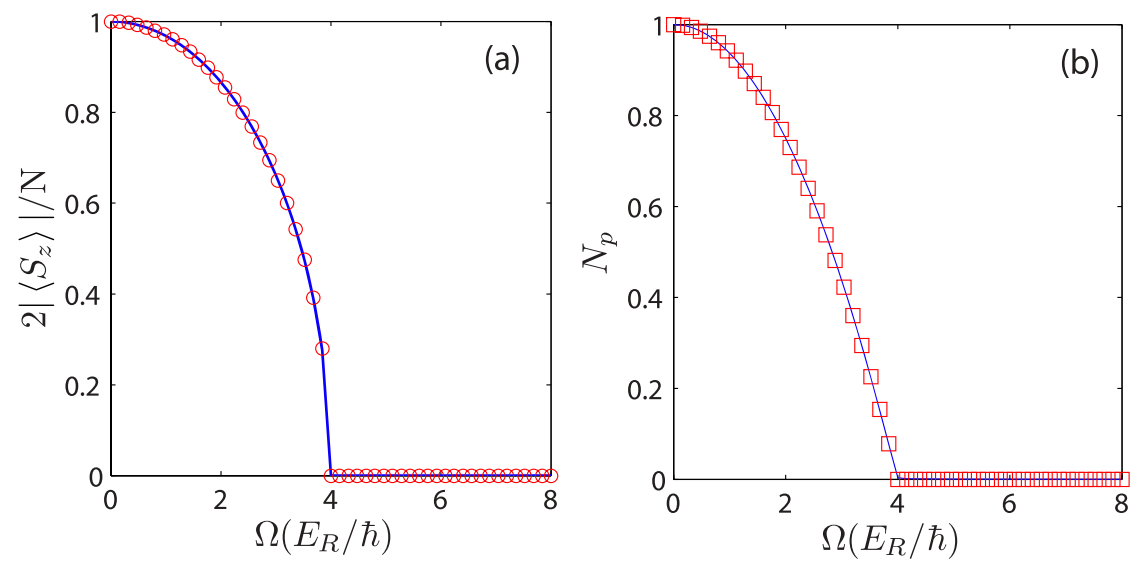

Figure $2 \mid$ Quantum phase transition in terms of spin polarization and occupation number. (a) Spin polarization and (b) occupation number of harmonic trap mode as a function of $\Omega$. The red squares are analytical results for the mean field and blue lines are the numerical results from the Hamiltonian (4). The parameters are chosen as $m=1.44 \times 10^{-25} \mathrm{~kg}, \lambda=804.1 \mathrm{~nm}, \omega_{x}=2 \pi \times 50 \mathrm{~Hz}$, and $N=4 \times 10^{3}$.

correlations. Spin squeezing can be quantified by the following parameter $^{39}$

$$
\xi^{2}=\frac{4 \min \left(\Delta S_{n_{\perp}}\right)^{2}}{N}
$$

with $\left(\Delta S_{n_{\perp}}\right)^{2}=\left\langle S_{n_{\perp}}^{2}\right\rangle-\left\langle S_{n_{\perp}}\right\rangle^{2}$. The subscript $n_{\perp}$ refers to an arbitrary axis perpendicular to the mean spin $S$. The inequality $\xi^{2}<1$ indicates the state is spin squeezed.

For the bosonic squeezing, it can be characterized by a parameter

$$
\zeta^{2}=\min _{\theta \in[0,2 \pi)}\left(\Delta X_{\theta}\right)^{2}
$$

where $X_{\theta}=X \cos \theta+P \sin \theta$ with $X=a+a^{\dagger}$ and $P=i\left(a^{\dagger}-a\right)$. For the special case, $\theta=\pi / 2, \zeta^{2}=\left(\Delta p_{x}\right)^{2}$, it means the field squeezing of the system corresponds to the momentum squeezing. For a quantum state, the field squeezing factor can also be written $\mathrm{as}^{54-56}$

$$
\zeta^{2}=1+2\left\langle a^{\dagger} a\right\rangle-2|\langle a\rangle|^{2}-2\left|\left\langle a^{2}\right\rangle-\langle a\rangle^{2}\right| .
$$

As is shown in Fig. 3, the spin and field squeezing factors as functions of $\Omega$ with $\delta=0$ are plotted. It is demonstrated that the spin squeezing parameter attains its minimal value at the boundary between the normal and superradiant phases. For the bosonic squeezing factor, it decreases as $\Omega$ increases, which indicates the system exhibits a bosonic squeezing when $\Omega \neq 0$.

For the above squeezing phenomenon, a natural question arises as how the system exhibits the spin and field squeezing. In Hamiltonian (4), we can not directly find the spin-spin interaction. In fact, both the spin and field squeezing can be induced by SOC. To demonstrate these arguments clearly, we employ a unitary transformation $U=\exp \left(i G\left(a^{\dagger}+a\right) S_{z}\right)$ with $G=\sqrt{2 m \gamma^{2} /\left(N^{2} \hbar \omega_{x}\right)}$ to rewrite Hamiltonian (4) as $H_{U}=U^{\dagger} H_{T} U^{17}$. After a straightforward calculation, we can obtain

$$
\begin{array}{r}
H_{U}=\hbar \omega_{x} N a^{\dagger} a-4 E_{R} / N S_{z}^{2} \\
+\hbar \Omega S_{x} \cos \left[G\left(a^{\dagger}+a\right)\right] \\
-\hbar \Omega S_{y} \sin \left[G\left(a^{\dagger}+a\right)\right] .
\end{array}
$$

For such a Hamiltonian, we can clearly see that the system shows the spin-spin interaction and nonlinear terms of harmonic trap mode from the terms $S_{z}^{2}$ and $\hbar \Omega S_{x} \cos \left[G\left(a^{\dagger}+a\right)\right]-\hbar \Omega S_{y}$ $\sin \left[G\left(a^{\dagger}+a\right)\right]$, respectively. Thus we can argue that the spin-orbit coupling induces the spin and field squeezing.
To derive an analytical result of spin squeezing factor, we need to reduce the terms $\hbar \Omega S_{x} \cos \left[G\left(a^{\dagger}+a\right)\right]-\hbar \Omega S_{y} \sin \left[G\left(a^{\dagger}+a\right)\right]$. For a large number of atom, we have $\max \left(\Omega, \gamma \sqrt{2 m \omega_{x} / \hbar}\right) \ll \omega_{x} N$ and thus $\left\langle a^{\dagger} a\right\rangle \ll N$. In fact the occupation number $\left\langle a^{\dagger} a\right\rangle$ of harmonic trap mode is inversely proportional to $\omega_{x} N^{16}$, which indicates the negligible term $\hbar \Omega S_{y} \quad \sin \left[G\left(a^{\dagger}+a\right)\right] \sim 0$ and the term $\hbar \Omega S_{x} \cos \left[G\left(a^{\dagger}+a\right)\right] \simeq \hbar \Omega S_{x}$. Then the Hamiltonian $H_{U}$ can be reduced to $H_{U}^{S}=-4 E_{R} / N S_{z}^{2}+\hbar \Omega S_{x}$, which corresponds to the Lipkin-Meshkov-Glick model. Treating the quantum effects as small fluctuations, an approximate result of $\xi^{2}$ can be obtained by using the Holstein-Primakoff transformation in the limit of large atom number. Introducing the Holstein-Primakoff transformation $\tilde{S}_{z}=N / 2-b^{\dagger} b$ and $\tilde{S}_{-}=\sqrt{N} b^{\dagger} \sqrt{1-b^{\dagger} b / N}=\left(\tilde{S}_{+}\right)^{\dagger}$ and using the Bogoliubov transformation $^{57,58}$, the Hamiltonian $H_{U}^{S}$ can be diagonalized and the spin squeezing parameter can be obtained as (see method)

$$
\xi^{2}=\left\{\begin{array}{l}
\sqrt{1-\left(\Omega / \Omega_{c}\right)^{2}}, \Omega<\Omega_{c}, \\
\sqrt{1-\Omega_{c} / \Omega}, \Omega>\Omega_{c} .
\end{array}\right.
$$

In the region $\Omega>\Omega_{c}$, when $\Omega \gg \Omega_{c}, \xi^{2} \simeq 1$. It is due to the fact that for the large value of $\Omega$, all the spins are polarized along the $x$ axis. In fact, $\Omega$ can be tuned from $10^{-2} \mathrm{kHz}$ to $\mathrm{MHz}$ in the current experiment, thus the system always exhibits spin squeezing. As is shown in Fig. 3(a), the numerical result agrees with the approximation analytical result in Eq. (10).

To obtain the result of the bosonic squeezing, we can reduce the Hamiltonian $H_{U}$ to an effective Hamiltonian (with the constant terms neglected)

$$
H_{U}^{B}=\hbar \omega_{x} N_{a}^{\dagger} a+u\left(a^{\dagger}+a\right)^{2}
$$

with $u=\frac{\hbar \Omega^{2}}{4 N \omega_{x}}$ and $\frac{\Omega E_{R}}{N \omega_{x}}$ for $\Omega<\Omega_{c}$ and $>\Omega_{c}$, respectively. In the above derivation, we have used $S_{x}=-N \hbar \Omega /\left(8 E_{R}\right)$ and $-N / 2$ for $\Omega$ $<\Omega_{c}$ and $>\Omega_{c}$, respectively. The Hamiltonian $H_{U}^{B}$ can be diagonalized by using the Bogoliubov transformation

$$
\tilde{a}=\cosh \sigma a-\sinh \sigma a^{\dagger}
$$

with $\tanh 2 \sigma={\frac{-2 u}{N \hbar \omega_{x}+2 u}}^{57,59}$. For the diagonalization of the Hamiltonian, we can find the ground state in the bosonic vacuum, 

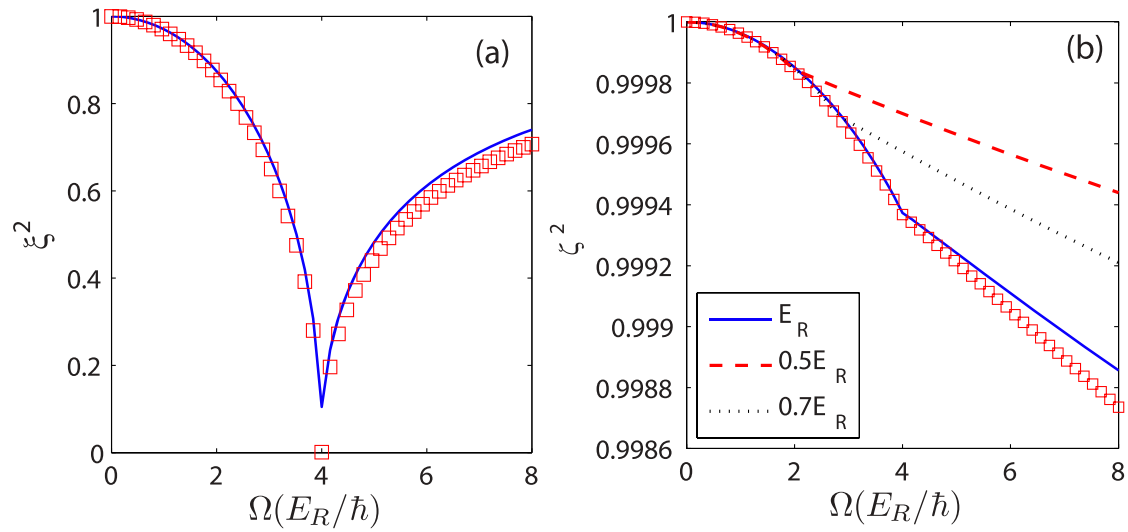

Figure $3 \mid$ Quantum phase transition in terms of spin and field squeezing parameters. (a) Spin squeezing parameter $\xi^{2}$ and (b) bosonic squeezing factor $\zeta^{2}$ as functions of $\Omega$. The red square denotes the analytical result in Eqs. (10) and (13), whereas the blue lines reflects the direct numerical results of Hamiltonian (4). The parameters are the same as those in Fig. 2.

i.e. $\left\langle\tilde{a}^{\dagger} \tilde{a}\right\rangle=0$, in terms of which the corresponding field squeezing parameter is given by

$$
\zeta^{2}=\left\{\begin{array}{l}
\frac{1}{\sqrt{1+\frac{\Omega^{2}}{\left(N \omega_{x}\right)^{2}}}}, \Omega<\Omega_{c}, \\
\frac{1}{\sqrt{1+\frac{4 \hbar \Omega E_{R}}{\left(N \hbar \omega_{x}\right)^{2}}}}, \Omega>\Omega_{c} .
\end{array}\right.
$$

We note that in the superradiant phase, $\zeta^{2}$ is independent of the SOC strength $\gamma$ which can be tuned through a fast and coherent modulation of Raman coupling ${ }^{16}$. As is shown in Fig. 3(b), $\zeta^{2}$ is plotted with different effective SOC strengths. We find the numerical results of Hamiltonian (4) coincide well with the analytical ones in Eq. (13).

Effect of detuning to spin and field squeezing. We proceed to consider the effect of the detuning on the spin polarization and the quasi-momentum. In a recent experiment, the spin polarization and quasi-momentum have been measured by linearly swept the detuning with different $\Omega^{18}$. It has been observed that the phenomenon of QPT vanishes and the BEC is always in the
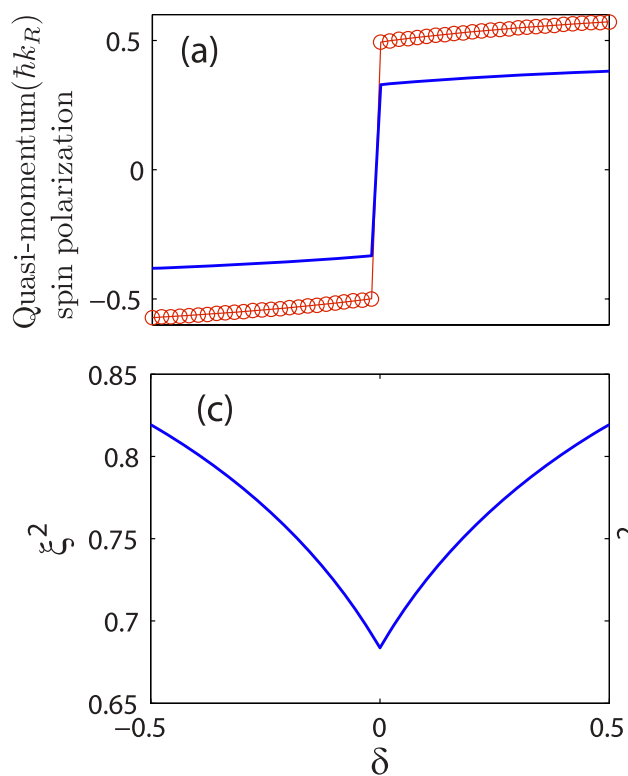

superradiant phase for a finite detuning. As is shown in Fig. 4(a), we investigate the ground state of the spin polarization and the quasimomentum with $\Omega=3 E_{R}$. We find that the spin polarization and the quasi-momentum exhibit a step jump at $\delta=0$ as the detuning $\delta$ increases. When $\delta<0$, most of the atoms are in the state $|\uparrow\rangle$, as $\delta$ increases and $\delta>0$, most of the ultracold atoms stay at the state $|\downarrow\rangle$. These numerical results coincide well with the experiment result ${ }^{18}$.

The occupation number of harmonic trap mode $N_{p}$ is also studied for the finite detuning. As is shown in Fig. $4(\mathrm{~b}), N_{p}$ is plotted as a function of the detuning. We can find that $N_{p}$ increases as the detuning $|\delta|$ increases, which means the presence of detuning increases the occupation number of harmonic trap mode. For the spin and field squeezing, shown in Fig. 4(c) and (d), $\xi^{2}$ and $\zeta^{2}$ increase as the value of $|\delta|$ increases, which indicates the presence of detuning suppresses the spin and field squeezing.

\section{Discussion}

We have investigated the spin-orbit coupled BEC via spin and field squeezing. By mapping the system to the well-known quantum Dicke model, we show that the quantum phase transition of superradiant (spin polarized)-normal (spin balanced) can be induced by tuning
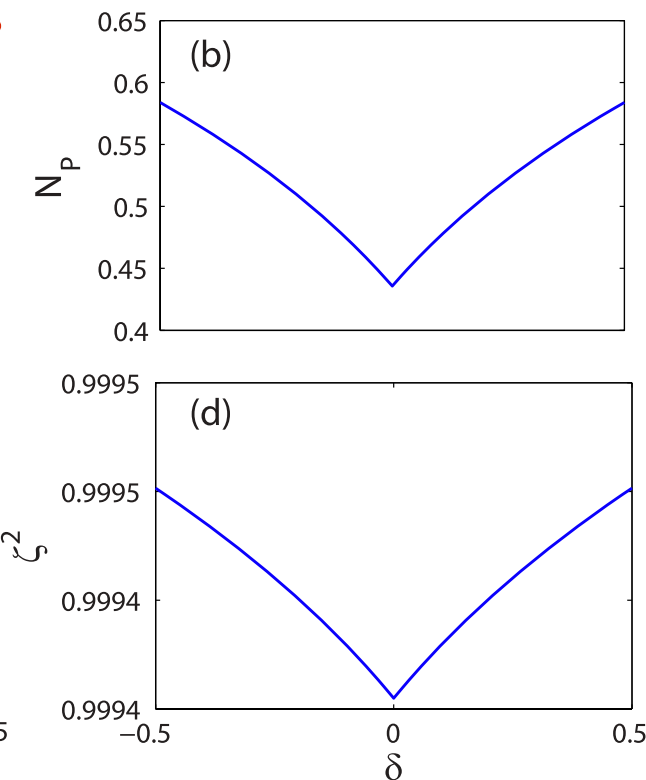

Figure $4 \mid$ Effect of the detuning. (a) Spin polarization (blue line) and quasi-momentum (red line with circles) (b) occupation number of harmonic trap mode $N_{p}$, (c) spin, and (d) field squeezing parameter as a function of Raman detuning $\delta$ with $\Omega=3 E_{R}$. The parameters are chosen as $m=1.44 \times$ $10^{-25} \mathrm{~kg}, \lambda=804.1 \mathrm{~nm}, \omega_{x}=2 \pi \times 100 \mathrm{~Hz}$, and $N=2 \times 10^{3}$. 
two Raman lasers with a current experimental setup of NIST. Crossing from the normal phase to the superradiant phase, macroscopic spin polarization and occupation number of harmonic trap mode emerge at the critical point. Therefore, the spin polarization and occupation number of harmonic trap mode can be treated as the order parameters revealing the quantum criticality. We further consider the behaviors of spin and field squeezing near the critical point and find that the spin and field squeezings reveal the quantum phase transition in different manners. At the critical point, the maximal spin squeezing (with the minimal squeezing factor) occurs and the field squeezing experiences a sudden transition. In addition, the field squeezing is independent of the SOC strength in the in the superradiant phase. Finally, the effects of the detuning on the ground state of spin polarization and quasimomentum are studied. The presence of the detuning suppresses the spin and field squeezing, while it enhances the occupation number of harmonic trap mode.

\section{Methods}

Here we employ the Holstein-Primakoff and Bogoliubov transformations to derive the result of spin squeezing. In the Sec. II, we obtain a reduced Hamiltonian $H_{U}^{S}=-4 E_{R} / N S_{z}^{2}+\hbar \Omega S_{x}$. For simplicity, we rewrite the form of $H_{U}^{S}$ as $\tilde{H}_{U}^{S}=-1 / N S_{x}^{2}-\hbar \Omega S_{z} / 4 E_{R}$ which indicates the spin direction along as $z$ axis. Rotating the $z$ axis to the semiclassical magnetization, we obtain ${ }^{57,58}$

$$
\left(\begin{array}{l}
S_{x} \\
S_{y} \\
S_{z}
\end{array}\right)=\left(\begin{array}{ccc}
\cos \theta_{0} & 0 & \sin \theta_{0} \\
0 & 1 & 0 \\
-\sin \theta_{0} & 0 & \cos \theta_{0}
\end{array}\right)\left(\begin{array}{c}
\tilde{S}_{x} \\
\tilde{S}_{y} \\
\tilde{S}_{z}
\end{array}\right),
$$

where $\theta_{0}=0$ for $\hbar \Omega / 4 E_{R}>1$ and $\theta_{0}=\arccos \left(\hbar \Omega / 4 E_{R}\right)$ for $\hbar \Omega / 4 E_{R} \leq 1$. Then the corresponding transformed Hamiltonian reads (with the constant terms dropped)

$$
\begin{aligned}
\tilde{H}_{U}^{\prime S} & =-\frac{\hbar \Omega}{2 E_{r}} m \tilde{S}_{z}-\frac{2}{N}\left[\left(1-m^{2}\right) \tilde{S}_{z}^{2}+m^{2}\left(\tilde{\mathbf{S}}^{2}-\tilde{S}_{z}\right) / 2\right] \\
& +\frac{\hbar \Omega}{4 E_{R}} \sqrt{1-m^{2}}\left(\tilde{S}_{+}+\tilde{S}_{-}\right)-\frac{m^{2}}{2 N}\left(\tilde{S}_{+}^{2}+\tilde{S}_{-}^{2}\right) \\
& -\frac{m \sqrt{1-m^{2}}}{N}\left(\tilde{S}_{+} \tilde{S}_{z}+\tilde{S}_{z} \tilde{S}_{+}+\tilde{S}_{-} \tilde{S}_{z}+\tilde{S}_{z} \tilde{S}_{-}\right),
\end{aligned}
$$

where $m=\cos \theta_{0}$. Introducing the Holstein-Primakoff representation

$$
\begin{aligned}
& \tilde{S}_{z}=N / 2-b^{\dagger} b, \\
& \tilde{S}_{-}=\sqrt{N} b^{\dagger} \sqrt{1-b^{\dagger} b / N}, \\
& \tilde{S}_{+}^{\dagger}=\sqrt{N} \sqrt{1-b^{\dagger} b / N b},
\end{aligned}
$$

where $b_{\uparrow}$ and $b$ satisfy the relation $\left[b, b^{\dagger}\right]=1$. Then we obtain the Hamiltonian (neglecting the constant terms)

$$
\begin{aligned}
& \tilde{H}_{U}^{\prime \prime S}=\left(1+m \hbar \Omega / 4 E_{R}-3 m^{2} / 2\right) b^{\dagger} b \\
& -m^{2}\left(b^{\dagger 2}+b^{2}\right) / 4
\end{aligned}
$$

up to the 0th order of $N$. Using the Bogoliubov transformation

$$
\tilde{b}^{\dagger}=\cosh (\theta / 2) b^{\dagger}+\sin (\theta / 2) b,
$$

the Hamiltonian $\tilde{H}_{U}^{\prime \prime}$ can be diagonalized with

$$
\tanh \theta=\frac{m^{2}}{2+m \hbar \Omega / 2 E_{R}-3 m^{2}} .
$$

When $\hbar \Omega / 4 E_{R}>1, m=1$, and we can obtain

$$
\begin{aligned}
& \left\langle S_{x}^{2}\right\rangle=\frac{N}{4} \sqrt{\frac{\hbar \Omega}{\hbar \Omega-4 E_{R}}}, \\
& \left\langle S_{y}^{2}\right\rangle=\frac{N}{4} \sqrt{\frac{\hbar \Omega-4 E_{R}}{\hbar \Omega}} .
\end{aligned}
$$

Then the corresponding spin squeezing parameter is given by

$$
\xi^{2}=\sqrt{1-\Omega_{c} / \Omega} .
$$

When $\hbar \Omega / 4 E_{R} \leq 1, m=h$, we have

$$
\begin{aligned}
\left\langle S_{x}^{2}\right\rangle & =\frac{\left(N^{2}+2 N\right)\left[1-\left(\hbar \Omega / 4 E_{R}\right)^{2}\right]}{4} \\
& +\frac{N^{2}-\left(2-\left(\hbar \Omega / 4 E_{R}\right)^{2}\right)\left(1-\left(\hbar \Omega / 4 E_{R}\right)^{2}\right)}{4 \sqrt{1-\left(\hbar \Omega / 4 E_{R}\right)^{2}}} .
\end{aligned}
$$

Then we obtain

$$
\xi^{2}=\sqrt{1-\left(\Omega / \Omega_{c}\right)^{2}}
$$

1. Nagaosa, N., Sinova, J., Onoda, S., MacDonald, A. H. \& Ong, N. P. Anomalous Hall effect. Rev. Mod. Phys. 82, 1539 (2010).

2. Xiao, D., Chang, M.-C. \& Niu, Q. Berry phase effects on electronic properties. Rev. Mod. Phys. 82, 1959 (2010).

3. Qi, X.-L. \& Zhang, S.-C. Topological insulators and superconductors. Rev. Mod. Phys. 83, 1057 (2011).

4. Žutić, I., Fabian, J. \& Das Sarma, S. Spintronics: fundamentals and applications. Rev. Mod. Phys. 76, 323 (2004).

5. Nayak, C., Simon, S. H., Stern, A., Freedman, M. \& Das Sarma, S. Non-Abelian anyons and topological quantum computation. Rev. Mod. Phys. 80, 1083 (2008).

6. Stanescu, T. D., Anderson, B. \& Galitski, V. Spin-orbit coupled Bose-Einstein condensates. Phys. Rev. A 78, 023616 (2008).

7. Lin, Y.-J., Jimenez-Garcia, K. \& Spielman, I. B. Spin-orbit-coupled Bose-Einstein condensates. Nature 471, 83 (2011).

8. Xu, X.-Q. \& Han, J. H. Spin-orbit coupled Bose-Einstein condensate under rotation. Phys. Rev. Lett. 107, 200401 (2011).

9. Yip, S.-K. Bose-Einstein condensation in the presence of artificial spin-orbit interaction. Phys. Rev. A 83, 043616 (2011).

10. Xu, Z. F., Lü, R. \& You, L. Emergent patterns in a spin-orbit-coupled spin-2 Bose Einstein condensate. Phys. Rev. A 83, 053602 (2011).

11. Zhou, X.-F., Zhou, J. \& Wu, C. Vortex structures of rotating spin-orbit-coupled Bose-Einstein condensates. Phys. Rev. A 84, 063624 (2011).

12. Kawakami, T., Mizushima, T. \& Machida, K. Textures of $F=2$ spinor BoseEinstein condensates with spin-orbit coupling. Phys. Rev. A 84, 011607(R) (2011).

13. Fu, Z., Wang, P., Chai, S., Huang, L. \& Zhang, J. Bose-Einstein condensate in a light-induced vector gauge potential using 1064-nm optical-dipole-trap lasers. Phys. Rev. A 84, 043609 (2011).

14. Zhu, Q., Zhang, C. \& Wu, B. Exotic superfluidity in spin-orbit coupled BoseEinstein condensates. EPL 100, 50003 (2012).

15. Zhang, Y., Mao, L. \& Zhang, C. Mean-field dynamics of spin-orbit coupled BoseEinstein condensates. Phys. Rev. Lett. 108, 035302 (2012).

16. Zhang, Y., Chen, G. \& Zhang, C. Tunable spin-orbit coupling and quantum phase transition in a trapped Bose-Einstein condensate. Sci. Rep. 3, 1937 (2013).

17. Lian, J., Yu, L., Liang, J.-Q., Chen, G. \& Jia, S. Orbit-induced spin squeezing in a spin-orbit coupled Bose-Einstein condensate. Sci. Rep. 3, 3166 (2013).

18. Hamner, C., Qu, C., Zhang, Y., Chang, J., Gong, M., Zhang, C. \& Engels, P. Dicketype phase transition in a spin-orbit-coupled BoseCEinstein condensate. Nature Commun. 5, 4023 (2014).

19. Li, Y., Pitaevskii, L. P. \& Stringari, S. Quantum tricriticality and phase transitions in spin-orbit coupled Bose-Einstein condensates. Phys. Rev. Lett. 108, 225301 (2012).

20. Lian, J., Zhang, Y., Liang, J.-Q., Ma, J., Chen, G. \& Jia, S. Thermodynamics of spinorbit-coupled Bose-Einstein condensates. Phys. Rev. A 86, 063620 (2012).

21. Ozawa, T. \& Baym, G. Stability of ultracold atomic Bose condensates with Rashba spin-orbit coupling against quantum and thermal fluctuations. Phys. Rev. Lett. 109, 025301 (2012).

22. Ozawa, T. \& Baym, G. Ground-state phases of ultracold bosons with RashbaDresselhaus spin-orbit coupling. Phys. Rev. A 85, 013612 (2012).

23. Kawakami, T., Mizushima, T., Nitta, M. \& Machida, K. Stable skyrmions in $S U(2)$ gauged Bose-Einstein condensates. Phys. Rev. Lett. 109, 015301 (2012).

24. Radić, J., Di Ciolo, A., Sun, K. \& Galitski, V. Exotic quantum spin models in spinorbit-coupled Mott insulators. Phys. Rev. Lett. 109, 085303 (2012).

25. Cole, W. S., Zhang, S., Paramekanti, A. \& Trivedi, N. Bose-Hubbard models with synthetic spin-orbit coupling: Mott insulators, spin textures, and superfluidity. Phys. Rev. Lett. 109, 085302 (2012).

26. Zhang, J.-Y. et al. Collective dipole oscillations of a spin-orbit coupled BoseEinstein condensate. Phys. Rev. Lett. 109, 115301 (2012).

27. Zhang, D.-W., Xue, Z.-Y., Yan, H., Wang, Z. D. \& Zhu, S.-L. Macroscopic Klein tunneling in spin-orbit-coupled Bose-Einstein condensates. Phys. Rev. A 85, 013628 (2012)

28. Zhang, D.-W., Fu, L.-B., Wang, Z. D. \& Zhu, S.-L. Josephson dynamics of a spinorbit-coupled Bose-Einstein condensate in a double-well potential. Phys. Rev. A 85, 043609 (2012).

29. Xu, Z. F., Kawaguchi, Y., You, L. \& Ueda, M. Symmetry classification of spinorbit-coupled spinor Bose-Einstein condensates. Phys. Rev. A 86, 033628 (2012).

30. Chen, G., Ma, J. \& Jia, S. Long-range superfluid order in trapped Bose-Einstein condensates with spin-orbit coupling. Phys. Rev. A 86, 045601 (2012). 
31. Zhang, Y. \& Zhang, C. Bose-Einstein condensates in spin-orbit-coupled optical lattices: flat bands and superfluidity. Phys. Rev. A 87, 023611 (2013).

32. Wang, C., Gao, C., Jian, C.-M. \& Zhai, H. Spin-orbit coupled spinor Bose-Einstein condensates. Phys. Rev. Lett. 105, 160403 (2010).

33. Ho, T.-L. \& Zhang, S. Bose-Einstein condensates with spin-orbit interaction. Phys. Rev. Lett. 107, 150403 (2011).

34. Zhang, Y., Mao, L. \& Zhang, C. Mean-field dynamics of spin-orbit coupled BoseEinstein condensates. Phys. Rev. Lett. 108, 035302 (2012)

35. Hu, H., Ramachandhran, B., Pu, H. \& Liu, X.-J. Spin-orbit coupled weakly interacting Bose-Einstein condensates in harmonic traps. Phys. Rev. Lett. 108, 010402 (2012).

36. Sinha, S., Nath, R. \& Santos, L. Trapped two-dimensional condensates with synthetic spin-orbit coupling. Phys. Rev. Lett. 107, 270401 (2011).

37. Zhang, C., Tewari, S., Lutchyn, R. M. \& Das Sarma, S. $p_{x}+i p_{y}$ Superfluid from swave interactions of fermionic cold atoms. Phys. Rev. Lett. 101, 160401 (2008).

38. Gong, M., Tewari, S. \& Zhang, C. BCS-BEC crossover and topological phase transition in 3D spin-orbit coupled degenerate Fermi gases. Phys. Rev. Lett. 107, 195303 (2011)

39. Kitagawa, M. \& Ueda, M. Squeezed spin states. Phys. Rev. A 47, 5138 (1993)

40. Wineland, D. J., Bollinger, J. J., Itano, W. M., Moore, F. L. \& Heinzen, D. J. Spin squeezing and reduced quantum noise in spectroscopy. Phys. Rev. A 46, 6797 (1992).

41. Wineland, D. J., Bollinger, J. J., Itano, W. M. \& Heinzen, D. J. Squeezed atomic states and projection noise in spectroscopy. Phys. Rev. A 50, 67 (1994).

42. Ma, J., Wang, X., Sun, C. P. \& Nori, F. Quantum spin squeezing. Phys. Rep. 509, 89 (2011)

43. Korbicz, J. K., Cirac, J. I. \& Lewenstein, M. Spin squeezing inequalities and entanglement of $N$ qubit States. Phys. Rev. Lett. 95, 120502 (2005).

44. Tóth, G., Knapp, C., Gühne, O. \& Briegel, H. J. Optimal spin squeezing inequalities detect bound entanglement in spin models. Phys. Rev. Lett. 99, 250405 (2007).

45. Guehne, O. \& Tóth, G. Entanglement detection. Phys. Rep. 474, 1 (2009).

46. Amico, L., Fazio, R., Osterloh, A. \& Vedral, V. Entanglement in many-body systems. Rev. Mod. Phys. 80, 517 (2008).

47. Horodecki, R., Horodecki, P., Horodecki, M. \& Horodecki, K. Quantum entanglement. Rev. Mod. Phys. 81, 865 (2009).

48. Wang, X. \& Sanders, B. C. Spin squeezing and pairwise entanglement for symmetric multiqubit states. Phys. Rev. A 68, 012101 (2003).

49. Takano, T., Fuyama, M., Namiki, R. \& Takahashi, Y. Spin squeezing of a cold atomic ensemble with the nuclear spin of one-half. Phys. Rev. Lett. 102, 033601 (2009).

50. Agarwal, G. S., Puri, R. R. \& Singh, R. P. Atomic Schrödinger cat states. Phys. Rev. A 56, 2249 (1997)

51. Deb, R. N., Sebawe Abdalla, M., Hassan, S. S. \& Nayak, N. Spin squeezing and entanglement in a dispersive cavity. Phys. Rev. A 73, 053817 (2006).
52. Chaudhury, S., Merkel, S., Herr, T., Silberfarb, A., Deutsch, I. H. \& Jessen, P. S. Quantum control of the hyperfine spin of a Cs atom ensemble. Phys. Rev. Lett. 99, 163002 (2007).

53. Dicke, R. H. Coherence in spontaneous radiation processes. Phys. Rev. 93, 99 (1954)

54. Lukš, A., Peřinová, V. \& Hradil, Z. Principal squeezing. Acta Phys. Pol. A 74, 713 (1988).

55. Alekseev, K. N. \& Prilmak, D. S. Squeezed states and quantum chaos. JETP 86, 61 (1998).

56. Bajer, J., Miranowicz, A. \& Tanaś, R. Limits of noise squeezing in Kerr effect. Czech. J. Phys. 52, 1313 (2002).

57. Dusuel, S. \& Vidal, J. Continuous unitary transformations and finite-size scaling exponents in the Lipkin-Meshkov-Glick model. Phys. Rev. B 71, 224420 (2005).

58. Ma, J. \& Wang, X. Fisher information and spin squeezing in the Lipkin-MeshkovGlick model. Phys. Rev. A 80, 012318 (2009).

59. Bakemeier, L., Alvermann, A. \& Fehske, H. Quantum phase transition in the Dicke model with critical and noncritical entanglement. Phys. Rev. A 85, 043821 (2012).

\section{Acknowledgments}

This work is supported by the Fundamental Research Funds for the Central Universities (Grant No. JUSRP11405), the Natural Science Foundation of Jiangsu Province of China (Grant No. BK20140128).

\section{Author contributions}

Y.H. wrote the main manuscript text and Z.-D.H. participated in the discussions. All authors reviewed the manuscript.

\section{Additional information}

Competing financial interests: The authors declare no competing financial interests. How to cite this article: Huang, Y. \& Hu, Z.-D. Spin and field squeezing in a spin-orbit coupled Bose-Einstein condensate. Sci. Rep. 5, 8006; DOI:10.1038/srep08006 (2015).

(i) (2) This work is licensed under a Creative Commons Attribution-NonCommercialShareAlike 4.0 International License. The images or other third party material in this article are included in the article's Creative Commons license, unless indicated otherwise in the credit line; if the material is not included under the Creative Commons license, users will need to obtain permission from the license holder in order to reproduce the material. To view a copy of this license, visit http:// creativecommons.org/licenses/by-nc-sa/4.0/ 